\section{Neural Correlates of Learning in the
Prefrontal Cortex of the Monkey: A \\ Neural Correlates of Learning in the
Prefrontal Cortex of the Monkey: A Predictive Model}

The principles underlying the organization and operation of the prefrontal cortex have been addressed by neural network modeling. The involvement of the prefrontal cortex in the temporal organization of behavior can be defined by processing units that switch between two stable states of activity (bistable behavior) in response to synaptic inputs. Long-term representation of programs requiring short-term memory can result from activity-dependent modifications of the synaptic transmission controlling the bistable behavior. After learning, the sustained activity of a given neuron represents the selective memorization of a past event, the selective anticipation of a future event, and the predictability of reinforcement. A simulated neural network illustrates the abilities of the model (1) to learn, via a natural step-by-step training protocol, the paradigmatic task (delayed response) used for testing prefrontal neurons in primates, (2) to display the same categories of neuronal activities, and (3) to predict how they change during learning. In agreement with experimental data, two main types of activity contribute to the adaptive properties of the network. The first is transient activity time-locked to events of the task and its profile remains constant during successive training stages. The second is sustained activity that undergoes nonmonotonic changes with changes in reward contingency that occur during the transition between stages.

Traditional views of cortical function define the posteroanterior dimension of the cortex as a substrate for the gradual transition from perceptual to motor processes, and then to organizational functions. Simple behavioral tasks have been used to assess this organization. A subject (rat, monkey, human) is asked to discriminate between or recognize visual, auditory, or somatosensory stimuli (sensory integration), to perform arm, eye, or body movements (motor performance), and more generally to produce a series of actions in response to environmental stimuli (temporal organization of behavior).

Although much remains to be understood, the mechanisms and cortical networks involved in sensory integration and motor performance have begun to be well characterized (Maunsell and Newsome, 1987; Johnson, 1992). Experimental studies of these functions also benefit from invaluable theoretical support, which defines the nature of the problems that must be solved by the brain.

The temporal organization of behavior has been far more difficult to deal with. Although convergent experimental evidence has demonstrated the critical role of the prefrontal cortex (PFC) in the acquisition and expression of complex behaviors (Goldman-Rakic, 1987; Fuster, 1988), the underlying information processing principles have not been identified. Theoretical studies have suggested that the PFC is involved in "working memory" (Goldman-Rakic, 1987), in "mediation of cross-temporal contingencies" (Fuster, 1988), and in "planning and problem solving" (Shallice, 1988). These definitions are attractive since they provide a formal, overall description of prefrontal functions. However, they provide no information on local mechanisms contributing to prefrontal functions.

These observations give rise to two main questions. (1) What is the specificity of information processing in the prefrontal cortex? The early work of Hubel and Wiesel (1968)
Emmanuel Guigon,' Bernadette Dorizzi, ${ }^{2}$ Yves Burnod,' and Wolfram Schultz ${ }^{3}$

1 INSERM CREARE, Université Pierre et Marie Curie, 75005 Paris, France, ${ }^{2}$ Département d'Informatique, Institut National des Télécommunications, 91011 Evry, France, and ${ }^{3}$ Institut de Physiologie, Université de Fribourg, CH-1700 Fribourg, Switzerland

described the mechanisms involved in processing sensory information (receptive field). Georgopoulos et al. (1982) have provided important insights into mechanisms of motor information processing (population code). Although these mechanisms are far from being precisely understood, they can help to understand experimental outcomes. However, no specific mechanisms have been identified, which may be responsible for processing in prefrontal circuits.

(2) How are sensory-motor programs represented in a neuronal network? We are now familiar with the ways visual objects may be stored in long-term memory (associative memories; Kohonen, 1988; Rolls, 1990) and with the way direction of movement is coded (Georgopoulos et al., 1986; Caminiti et al., 1991; Burnod et al., 1992). But what is the neuronal representation of a temporal sequence of events?

This article addresses these issues using a computational model. The modeling framework is connectionist (Hertz et al., 1991) in the sense that the model is built by asking questions about representation of information, connectivity, neural processing, and plasticity. However, we have made no a priori choices and the main features of the model are based on the principles of organization and operation in the PFC. The properties of the model are illustrated by computer simulations, which show the behavior of the network when it is trained to execute a spatial delayed response (DR) task. This task has been chosen because it has been well documented in nonhuman primates by a number of neurophysiological and neuropsychological studies and because it appears to strongly challenge prefrontal functions for both learning and executing the task (Jacobsen, 1935). The results concern both the execution of the task and the learning session (modulation of activity)

\section{Computational Properties of the Prefrontal Cortex}

\section{Sustained Activities in the Prefrontal Cortex}

A striking feature of prefrontal neuronal operations is the pattern of sustained activity observed during the delay imposed between a sensory cue and the production of a response to it (Fuster, 1973; Niki and Watanabe, 1976). Both experimental and theoretical studies have stressed the cardinal role of long-lasting activities. Electrophysiological recordings in behaving monkeys indicate that long-lasting activities are strongly correlated with behavioral processes, such as short-term memorization of instruction cues, expectation of forthcoming signals, and preparation of a behavioral reaction. Theoretical approaches have emphasized the contribution of sustained activities to unifying functions such as "working memory" (Goldman-Rakic, 1987) or "mediation of cross-temporal contingencies" (Fuster, 1988)

Sustained activities in the prefrontal cortex have three important characteristics. First, whatever the modalities used (visual or auditory cues, arm or eye movement responses), they occur during the delay between an instruction cue and the final permission to use the information contained therein for producing a response. Second, the duration of the activity is linked to the duration of the delay. Increasing the delay's length 


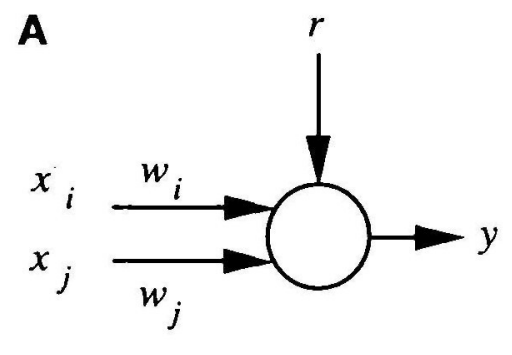

B

C

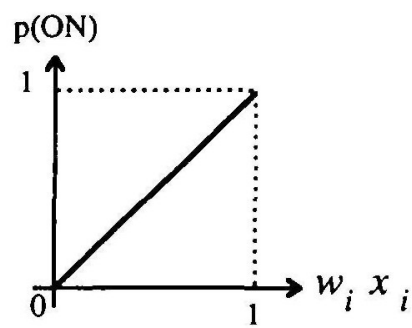

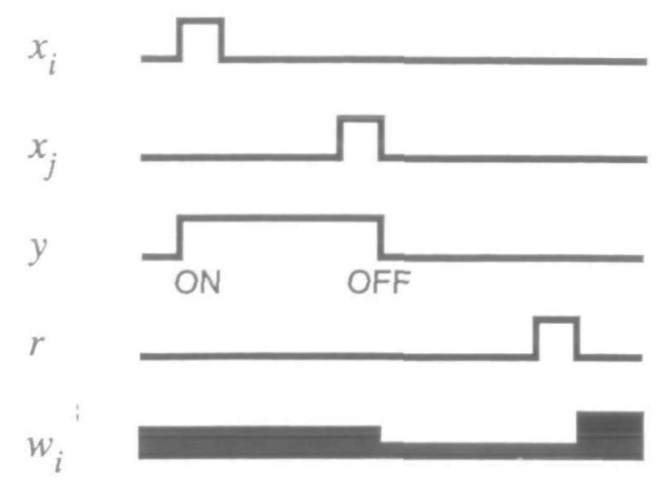

Figure 1. The properties of the bistable rule. $A$, The unit has two weighted input pathways $x_{i}(w)$ and $x_{j}\left(w_{j}\right)$, a reinforcement pathway $r$, and an output pathway $y$. $B, 0$ ualitative

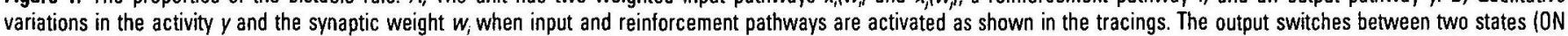
and OFF) for synaptic inputs. Note the conditions that elicit change in the synaptic weight: a decrease with the end of the sustained activity and increase with reinforcement. $C$, Transition functions indicating the probability of a switch between the two states $[p(O N)$ and $p(O F F)$. The corresponding equations are given in the Appendix.

leads to a prolonging of the activity (Kojima and GoldmanRakic, 1982; Batuev et al., 1985). Third, these activities are a product of learning (Fuster, 1973; Kubota and Komatsu, 1985). Recording in untrained animals, Fuster (1973) found fewer delay-related activities than in trained monkeys in similar behavioral conditions. There appears to be a relationship between the amount of delay activation and the level of performance (Fuster, 1973; Watanabe, 1986).

On the basis of these observations, we therefore propose that prefrontal neurons have two stable states of activity (bistable), and that transitions between these states are elicited by synaptic inputs. We also propose that this bistable behavior is controlled by learning and allows sensorimotor sequences to be built up under the control of a reinforcement signal.

The first part of this proposal is consistent with the fact that neurons can generate precisely controlled bistable state activity either in a single cell (Kiehn, 1991) or in a local recurrent circuit (see Kirillov et al., 1993). The present model makes no a priori assumption about the origin of the bistable behavior; the possible origin of a bistable behavior will be addressed in the Discussion.

The second part is new since it postulates that the plasticity of prefrontal neurons is produced by sustained activities and reinforcement. It implies that long-term changes in these neurons should take into account the temporal dimension defined by long-lasting discharges. However, most rules for activity-dependent modifications of synaptic transmission are based on the principle of temporal contiguity proposed by Hebb (1949) and result in a spatial competition between inputs (Stent, 1973). These rules may not be appropriate in the temporal domain. Sutton and Barto (1981) have proposed a simple way to extend the Hebbian rule by combining "traces" of inputs with postsynaptic activity. Other mechanisms, such as the timevarying threshold between the increase and decrease of synaptic efficacy, result in a specific temporal integration of successive events (Bienenstock et al., 1982). Based on these models, we propose a new rule (bistable rule) that combines bistable behavior and reinforcement.

The nature of the reinforcement signal is also important, as it seems to play a critical role in the PFC. This role is charac- terized by the inability of animals lacking a PFC to adjust their behavior to cope with changes in reward contingencies (Fuster, 1988). Multiple diffuse modulatory systems innervate the PFC and can deliver large-scale unconditional influences that are correlated with the reinforcing value of external events (Ljungberg et al., 1992).

\section{Bistable Rule}

The translation of our proposal into computational terms is illustrated in Figure 1. A neuron-like element, with two of its weighted input pathways $x_{i}\left(w_{i}\right)$ and $x_{j}\left(w_{j}\right)$, an output pathway $y$, and a reinforcement pathway $r$, is taken as a model of prefrontal neurons (Fig. $1 A$ ). Inputs, output, and reinforcement are binary variables, while synaptic weights can vary in the interval $[0,1]$. The behavior of the neuron when the two inputs $i$ and $j$ are successively stimulated is shown in Figure $1 B$. Unlike classical neural automata (McCulloch and Pitts, 1943), which display transient responses to transient inputs, the present neuron remains activated (state $O N$ ) for some time after the input $i$. The neuron then returns to rest after the second input $j$ (state OFF).

Transition functions are shown in Figure 1C. Transition to the ON state follows a classical law used to model the stochastic behavior of neurons (Sejnowski, 1986): the probability of transition is proportional to the summed inputs. Transition to the OFF state has two components: a spontaneous transition with a fixed probability $\eta$, and an unconditional transition following subsequent inputs. The spontaneous transition may be viewed as an effect of noise. In this formulation, we have assumed that only the transition to the ON state (sustained activity) is controlled by a synaptic weight. The neural network model of the following section will show that this assumption is sufficient to learn temporal sequences of events.

The second part of the proposal concerns long-term changes in synaptic weights depending on the bistable behavior. The rule is illustrated in Figure $1 B$. The synaptic weight, which controls the ON transition, first undergoes a decrease at the transition to the OFF state and then a greater increase after the reinforcement signal. This rule results in a global increase for reinforced sequences and a global decrease for nonreinfor- 
ced sequences. Before learning, synaptic weights are assumed to have low values, and thus units are unlikely to become activated (sustained activity). Repeated presentations of a reinforced sequence lead to increased weight. Thus, after learning, the unit will become activated when the first event of the sequence is presented, predicting the occurrence of a reinforcement. This rule relates the quantity of sustained activity to the predictability of reinforcement. A mathematical description of the model is given in the Appendix.

The following section illustrates the properties of the bistable rule for a network. This rule allows a DR task to be learned and neuronal activity in prefrontal neurons during acquisition of this task to be predicted.

\section{A Neural Network Model of Prefrontal Circuits}

A neural network model, which illustrates the properties of the bistable rule, was designed according to the principles of organization of prefrontal connections and was trained to execute a spatial DR task, which is a task generally used to study prefrontal neuronal activities in behaving monkeys.

\section{Task and Training}

The task involves two lights mounted above two horizontally arranged levers and a trigger light. At each trial, one of the two lights comes on for a short period. A few seconds after the instruction is extinguished, the trigger light comes on and the animal touches the lever indicated by the instruction; it receives a drop of liquid as a reward. The light provides information as to where to touch (instruction stimulus), whereas the trigger only determines the moment of touching (go signal). This task requires the spatial information contained in the instruction to be memorized for a short period.

Animals learn a DR task by a protocol of at least three stages. Initially, they learn to touch one of the two levers (movement) whenever they wish, and receive a drop of liquid (reinforcement). The lever becomes associated with a primary liquid reward and constitutes a conditioned incentive stimulus (drive), which, based on the thirst drive of fluid-deprived animals, creates an internal motivational state eliciting the movement (Toates, 1986). In the next stage, the animal is only allowed to touch a lever after a trigger light (go signal) has come on. This light now constitutes the incentive stimulus and determines the time of touching. In the final stage, the instruction light (instruction stimulus) comes on before the trigger light and determines which lever is to be touched after the trigger light. Although this three-stage protocol is much simpler than real laboratory training, it provides a functional description of the major behavioral changes that occur during the training in monkeys.

The training protocol of the model contained three stages $\left(1,1^{\prime}+2,2^{\prime}+3\right)$, where stages 1,2 , and 3 were those described above, and stages $1^{\prime}$ and $2^{\prime}$ were intermediary stages allowing the transition between successive behaviors.

\section{Network Arcbitecture}

The PFC has been described as a region of cross-modal integration (Jones, 1969; Barbas and Mesulam, 1985; for a review, see Fuster, 1988). It especially receives afferents from and projects to the major association cortices. (1) Visual information related to discrimination and recognition can be provided by the higher processing steps of the temporal lobe. The inferotemporal cortex projects directly to prefrontal areas, particularly to the ventral convexity (Jones and Powell, 1970; Ungerleider et al., 1989). Integrated information on the properties of visual stimuli are thus likely to be provided to PFC circuits. (2) Information relative to spatial location originates in the posterior parietal areas (Mishkin et al., 1983). There are major connections between these structures and the PFC (Petrides and
Pandya, 1984; Cavada and Goldman-Rakic, 1989). Strong interactions also exist between the prefrontal areas and structures involved in motor control (motor areas, basal ganglia; Alexander et al., 1986; Barbas and Pandya, 1987).

Note that the PFC is not connected to the primary sensory and motor areas, but has direct access to higher-order representations within all modalities.

The PFC also receives important projections from the hypothalamus, the amygdala, and several brainstem structures, directly or indirectly through the thalamus (reviewed in Fuster, 1988). These limbic structures are involved in the control of behavior (review in McGinty and Szymusiak, 1988) and can inform the PFC about drive and reinforcement. Fuster (1988) has emphasized the importance of a "drive" signal for the acquisition and execution of goal-directed behaviors. The PFC appears to need such a signal, which may be available from these limbic structures, to search actively for new, more suitable behaviors.

We have already stressed that a reinforcement signal is available to prefrontal circuits. In the model, this signal is considered to have an unconditional influence that depends on the correctness of the behavior.

These observations are summarized in the network architecture of Figure 2. The network is made of an input layer (sensory), an output layer (motor), and two hidden layers: (1) a matching layer in which units model neurons in higher-order sensory and motor regions and (2) a bistable layer in which units are models of prefrontal neurons. Their function has been described in the previous section (bistable rule).

The sensory layer consists of units coding for task events (instruction stimuli, go signal, lever position). Each sensory unit signals for the occurrence of a particular event by an all-ornone code. In the same way, movements toward the levers are coded in the output layer. The sensory layer projects divergently to the matching layer in such a way that multiple representations of the same sensory input can be found in the matching layer. Units related to lever position in the matching layer project to movement-related units in the output layer, implementing a direct relationship between lever position and movement toward the lever. Each matching unit is reciprocally connected to a group of units (squares; a group is four units) in the bistable layer and sends nonreciprocal connections to some other groups of bistable units. Bistable units have modifiable synapses that allow the network to form internal representations of temporal contingencies in the environment. Bistable units are also connected to two specific pathways: a drive pathway made active at the beginning of each behavior of the network, and a reinforcement pathway activated when a correct behavior is produced by the network.

As illustrated in Figure 2, matching units combine two sources of information. We have assumed that matching units perform a multiplicative (or gating) operation between the two types of input. In this way, the tuning of matching units to particular events is modulated by bistable inputs reflecting internal representations. The form of this combination was chosen based on recent experimental and theoretical observations in higher-order sensory and motor areas. Multiplicative combinations have been observed between eye position and retinal position in the primary visual cortex (Trotter et al., 1992) and in the posterior parietal cortex (Anderson et al., 1987), between vision and memory in the inferotemporal cortex (Eskandar et al., 1992a,b), and between arm position and visual trajectory in the motor and premotor cortex (Caminiti et al., 1991; Burnod et al., 1992). The biological basis for neuronal multiplication is discussed by Burnod et al. (1992) and Eskandar et al. (1992b).

The connectivity of the network defines multiple interactions between matching and bistable units, but does not cor- 
Figure 2. Network architecture. The matching layer is made of eight units. The bistable layer is made of eight groups of four units. Notations for the task events are as follows: Heft and l-right, positions of left and right levers; $m$-left and $m$-right, movements toward the levers; $g s$, go signal; $;$-left and i-right, instruction stimuli; $\delta$ and $r$, drive and re inforcement, respectively. We have made the following assumptions: (1) a redundant representation is used when the same event occurs in different situations (go signal); (2) all the units in a given bistable group have the same relationships with matching units; (3) there are no interfering events, except during the first training stage, where four movements (m-left, m-right, $m$-up, m-down) toward four different levers (1-left, I-right, 1-up, 1-down) are possible (but only two are correct). During this stage, the network learns to suppress the responses $m$-up and $m$-down when presented with I-up and I-down, respectively. It illustrates the ability of the network to deal with environmental interferences. Interfering events could be added at other training stages in a similar way.

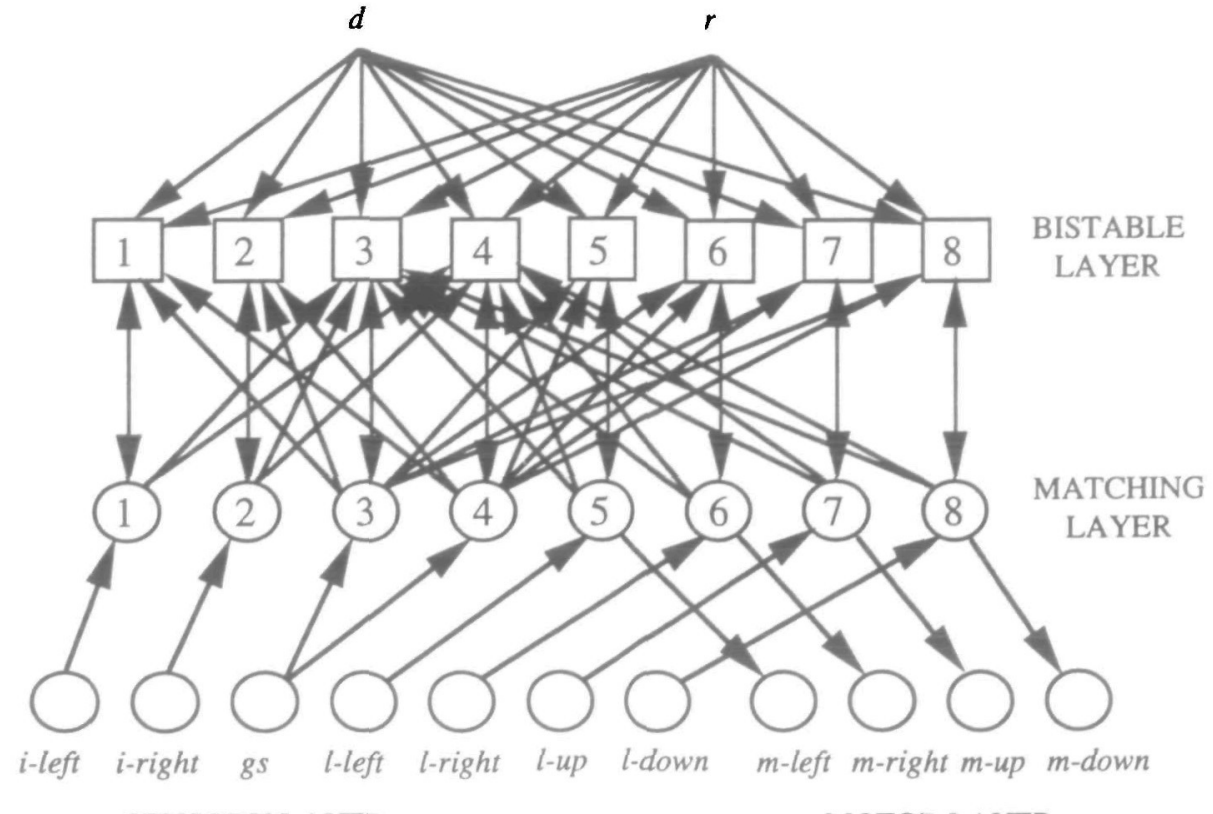

SENSORY LAYER
MOTOR LAYER respond to an a priori representation of particular functions. The range of possible behaviors of the network depends on the information available to matching units through input and output layers and on the ability of bistable units to learn relationships between sensory and motor activations.

\section{Computer Simulations}

We have performed computer simulations of the neural network described above. The network was trained in successive stages. At each stage, the network went through a number of trials, until the required behavior was reached. Each trial began with the delivery of the drive signal and ended with the reinforcement signal when the behavior was correct. A delay of length $\delta$ (measured in relation to the simulation time step) was introduced between the instruction stimulus and the go signal in the stage 3.

The results were collected as follows. At each training stage $\left(1,1^{\prime}+2,2^{\prime}+3\right)$, we examined the network during the execution of a trial. We noted whether the trial was correct and recorded the neuronal activities during the trial (units' output). Individual trial data were collected for each stage by a block of correct or erroneous trials to compute cumulative histo- grams of activity. Individual trial data were collected by block of trials to compute the performance rate during the learning of the task. Cumulative histograms were collected to compute the variation in block activity during the learning of the task. Simulations are described in the Appendix.

\section{Results}

This section provides a quantitative evaluation of our model. The results concern the global behavior of the network (as measured by the number of correct trials) and the behavior of units in the network (measured by units output activation), both during the training and once the training has been completed. The behavior of the network can be compared with the behavior of a monkey, while unit activation can be compared with available data on neuronal activity in the cortex of behaving monkeys.

\section{General Bebavior of the Network}

The performance of the network, as obtained by computer simulations, is shown in Figure 3: the number of correct trials increased during the acquisition of a particular behavior and decreased when behavior was changed. The random behavior
Figure 3. Computer simulations of network performance. Vertical dashed lines delimit training stages $11,1^{\prime}+2,2^{\prime}+$ 3). Each division on the horizontal axis corresponds to a block of eight successive trials. The corresponding ordinate value is the proportion of reinforced trials in the block. Note that stage 1 corresponds to the selection of two movements out of four. Initial performance is thus random. Parameter values are given in the Appendix.

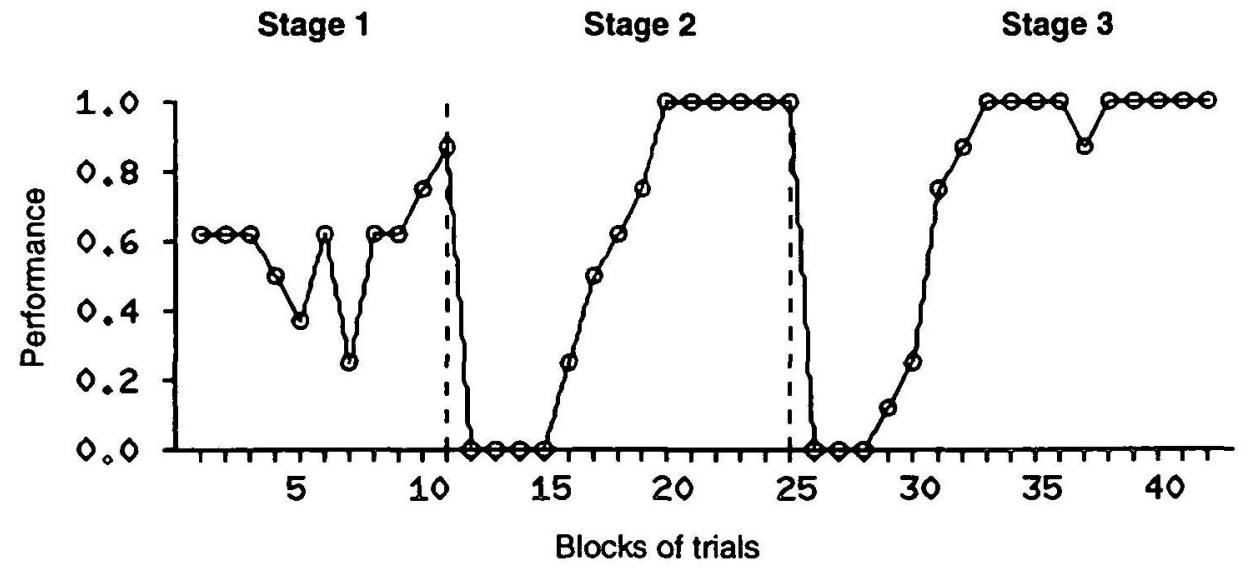



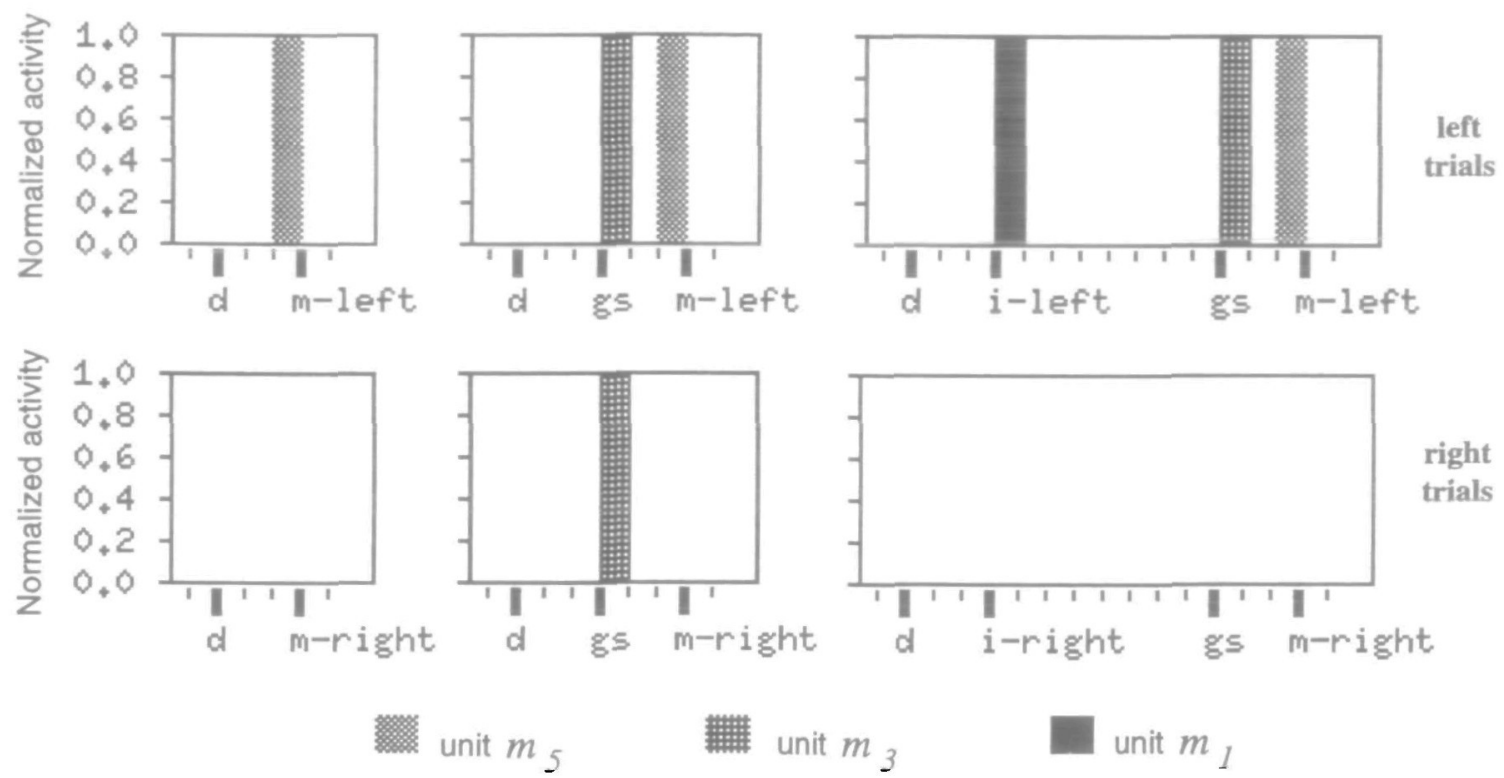

unit $m_{3}$

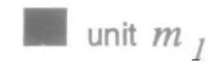

Figure 4. Evolution of output activity of three matching units during the training stages (left to right, Stages 1-3) for left and right trials. Each histogram shows the mean activity of the unit in 15 successive reinforced (left or right) trials, from just before the drive to just after the movement. The horizontal axis is time. Horizontal divisions (thin vertical lines) correspond to the simulation step (1 division $=1$ iteration). Task events are indicated with thick vertical lines. The reinforcement event is not shown. The mean normalized activity (percentage of trials during which the unit is active) is displayed on the vertical axis. Unit $m_{5}$ displayed a transient activity (one time step) before the leftward and rightward movements, respectively, in the three training stages. Unit $m_{3}$ was active with the go signal (gs) at stage 2 , and was preferentially active for left trials at stage 3 . Unit $m_{1}$ became active at stage 3 and displayed differential activity for left and right instruction stimuli. Same notations as Figure 2.

at the beginning of the first stage is explained by the choice of two movements out of four. After completing the training, the network was able to produce the correct behavior when presented with a set of events corresponding to a DR task trial. The global behavior of the network thus qualitatively reflected the behavior of a monkey during similar training.

\section{Activities after Learning}

Figures 4 and 5 illustrate the activities of different units in the network (Fig. 2) during the successive stages of training. The patterns of activity can be divided into several categories (anticipatory, stimulus-related, delay-related, movement-related), according to the temporal relationships between task events and peaks of activity. Different patterns occurred in matching and bistable units.

\section{Matching Units}

Matching units displayed transient activities that were timelocked to the onset of events (Fig. 4). Unit $m_{5}$ was active just before the movement, and was differentially active for left versus right movements. Similar results were obtained for unit $m_{6}$, which was preferentially related to rightward trials (not shown). Unit $m_{3}$ was active following the go signal, and unit $m_{1}$ was related to the instruction stimulus. These types of activity have been observed experimentally. Transient activities related to instruction stimuli or go signal have been found in the inferotemporal cortex (Mikami and Kubota, 1980; Fuster et al., 1985), and related to instruction stimuli, go signal, or movements in motor and premotor areas (Alexander and Crutcher, 1990; di Pellegrino and Wise, 1991).

\section{Bistable Units}

All the units within each bistable group displayed similar patterns of activity (not shown). Bistable units of different groups provided different patterns of activity, according to their connections to matching units (Fig. 5). The most interesting one is differential delay activity; this was a sustained activity between the onset of the instruction stimulus and the onset of the go signal specific for right versus left trials (group $b_{3}$; Fig. $5 B$, Stage 3 ). Other patterns included differential anticipatory activity before the movement (group $b_{5} ;$ Fig. $5 A$, Stage 3 ) and before the instruction (group $b_{1}$; Fig. $5 C$, Stage 3 ).

All these patterns have been described in the PFC during the performance of DR tasks (Fuster, 1973; Niki and Watanabe, 1976; Komatsu, 1982; see also, for oculomotor paradigms, Funahashi et al., 1990). These results thus appear to be consistent with experimental data. Other results concern learning-dependent changes in activity of the processing units. Since there have been few studies on neuronal activity during learning, these results can be considered as predictions of the model.

\section{Modulation of Activity during Learning}

The model predicts the changes in neuronal activities that will occur in the course of learning. Unit outputs are modulated according to the dynamics of processing and activity-dependent variations of synaptic weights. The changes concern not only the level of activity, but also the temporal pattern and the relation to external events.

\section{Matching Units}

Figure 4 shows the evolution of the pattern of activity for three matching units. The units displayed transient activity (one time step of the simulation) that was time-locked to sensory or motor events (movement in unit $m_{5}$, go signal in unit $m_{3}$, and instruction stimulus in unit $\boldsymbol{m}_{\mathbf{1}}$ ). Units became active when a given sensory or motor event was introduced (at stage 1 in $m_{5}$, at stage 2 in $m_{3}$, and at stage 3 in $m_{1}$ ).

Activity in unit $m_{5}$ was related to the movement toward the left lever. There was activity before the onset of the movement in the three training stages. This unit retained the same temporal pattern of activity and the same relationship with external events during the different learning stages. Unit $m_{3}$ became 
A group $b_{5}$
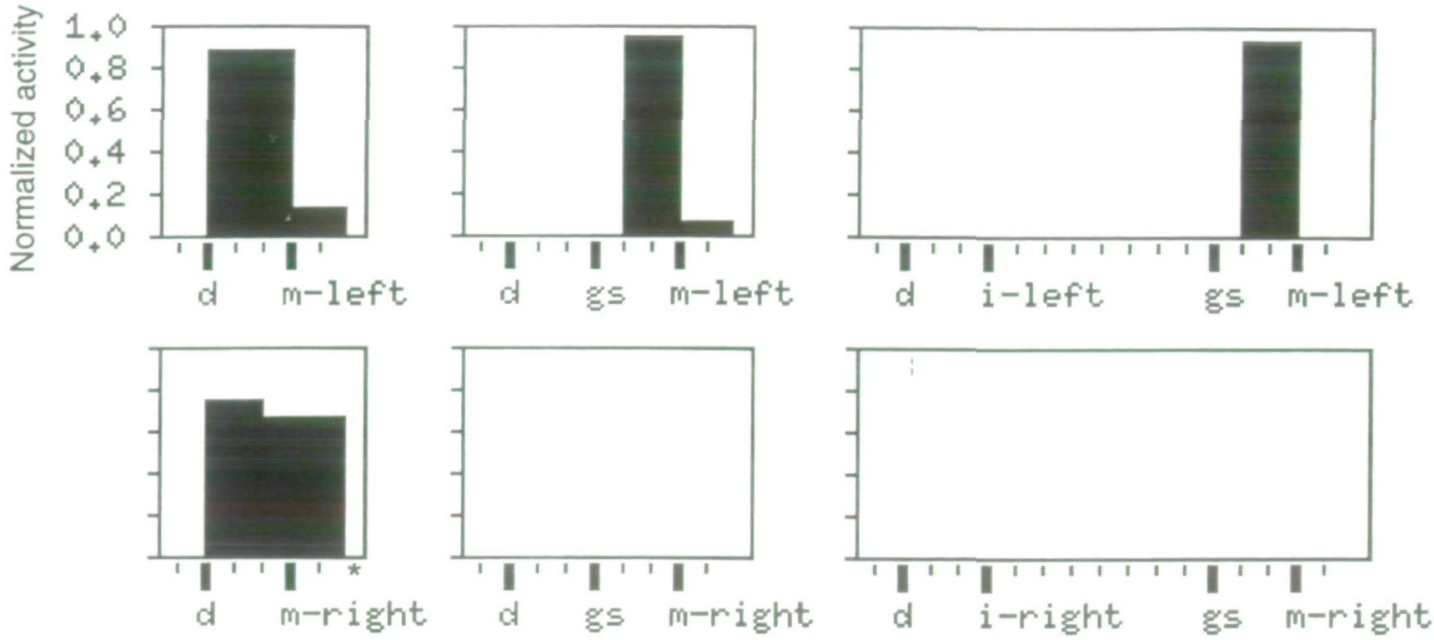

right

trials

B group $b_{3}$
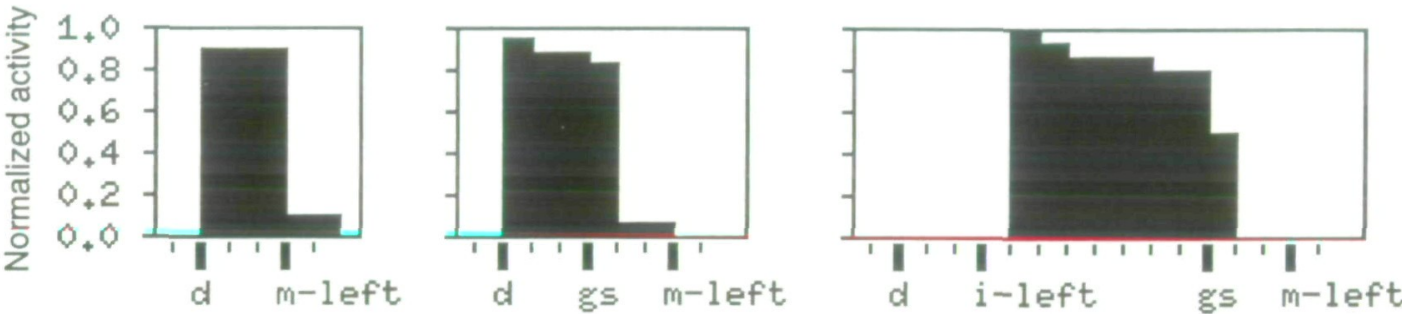

left

trials
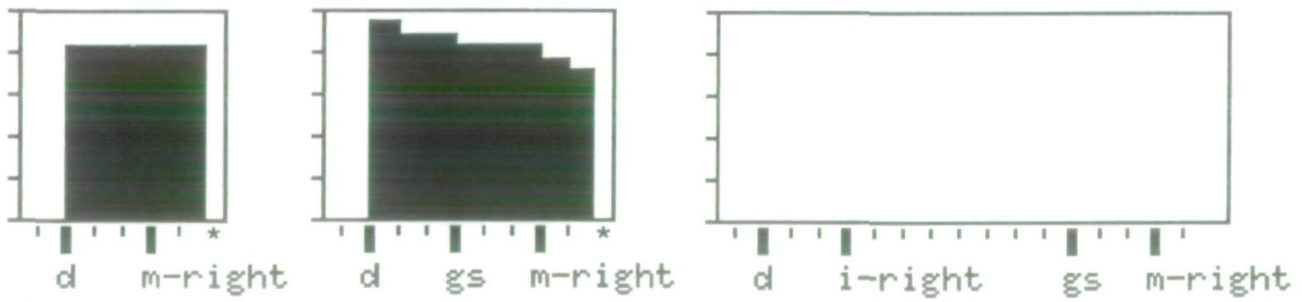

right

trials

C group $b$,
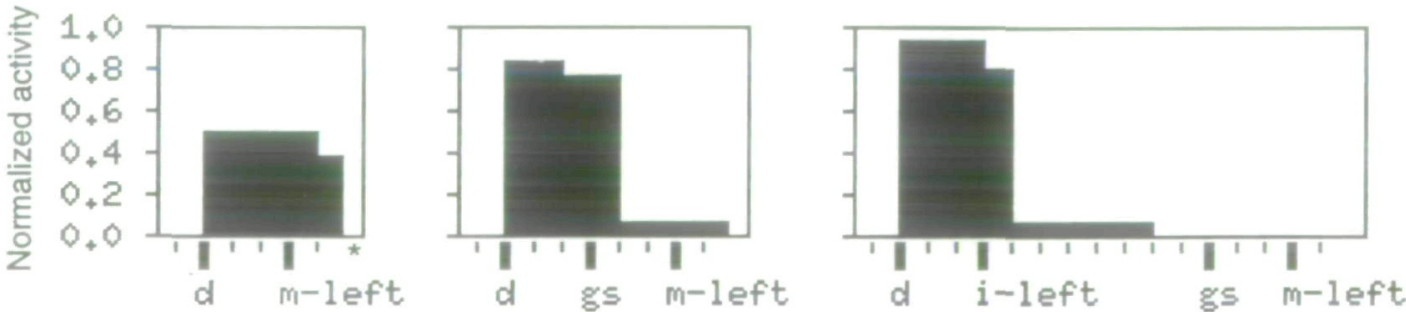

left

trials
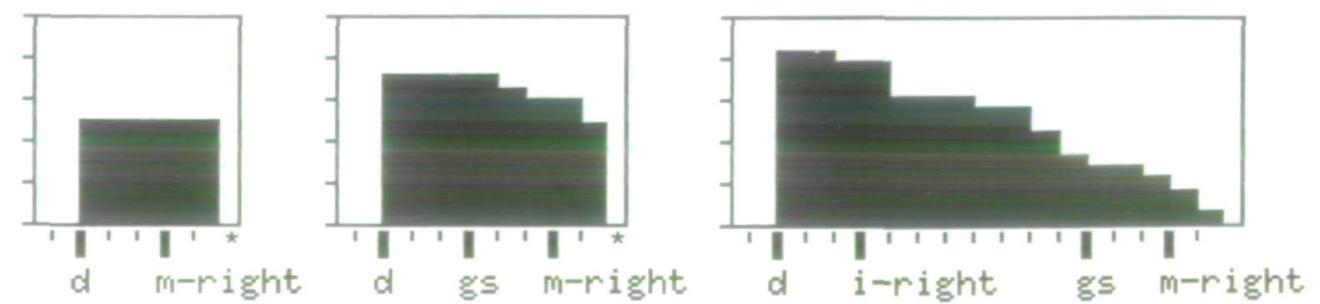

right

trials

Figure 5. Evolution of the output activity of three bistable units (top to bottom, $A-C$ ) during the different training stages (left to right, Stages $1-3)$. Unit $b_{5}(A)$ was sustainedly active (more than two time steps) before left movements in all three training stages. Activity ended with the movement. Unit $b_{3}(B)$ was sustainedly active in all three training stages. between the drive and the movement at stage 1, between the drive and the go signal at stage 2, and during the delay between the instruction stimulus and the go signal at stage 3. Activity was different for left and right trials. Unit $b,(C)$ was sustainedly active in anticipation of task events, at stages 2 and 3 , differentially for left and right trials. Activity started with the drive, but stopped with the go signal at stage 2 . and with the instruction stimulus at stage 3 . Histograms are the same as in Figure 4 . The asterisk $(*)$ indicates that sustained activity continues after the movement, but is not shown on the histogram. Same notations as Figure 2. 
$\begin{array}{llll}A \text { group } b_{5} & \text { Stage } 1 & \text { Stage } 2 & \text { Stage } 3\end{array}$

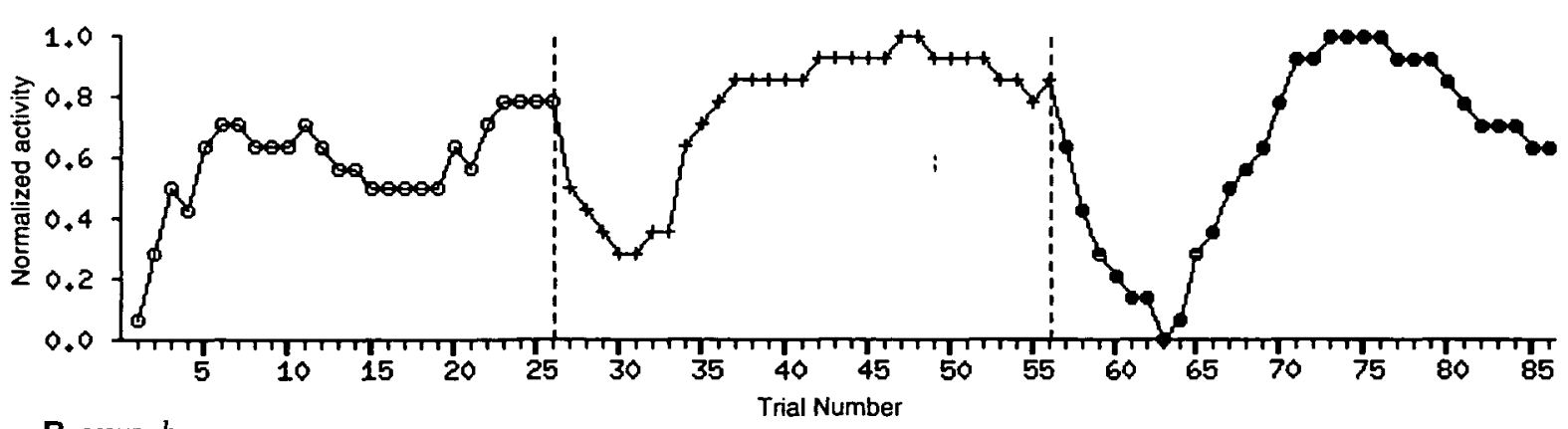

B group $b_{3}$

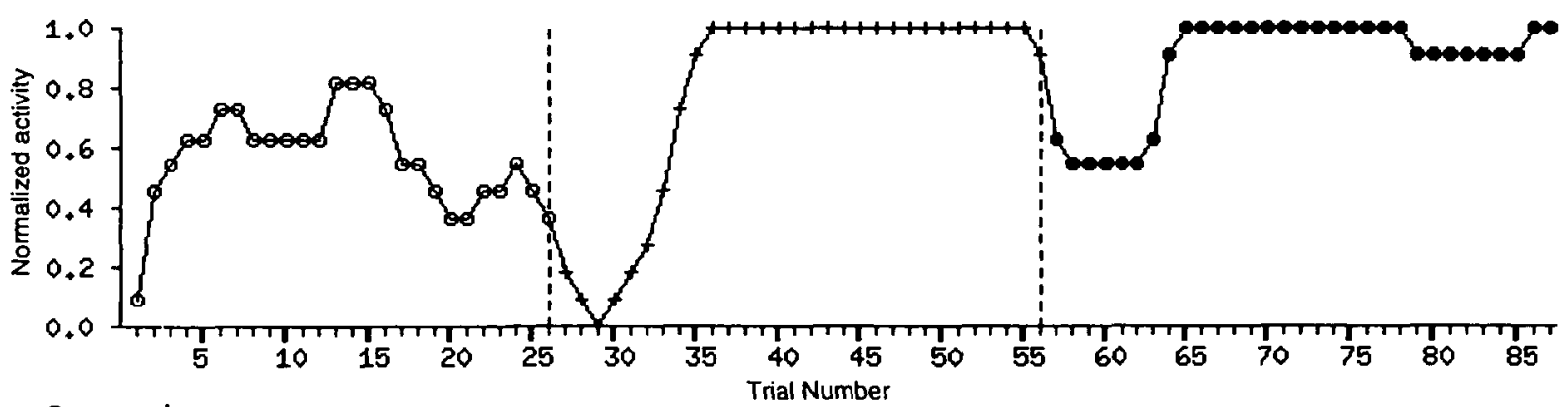

$C$ group $b$,

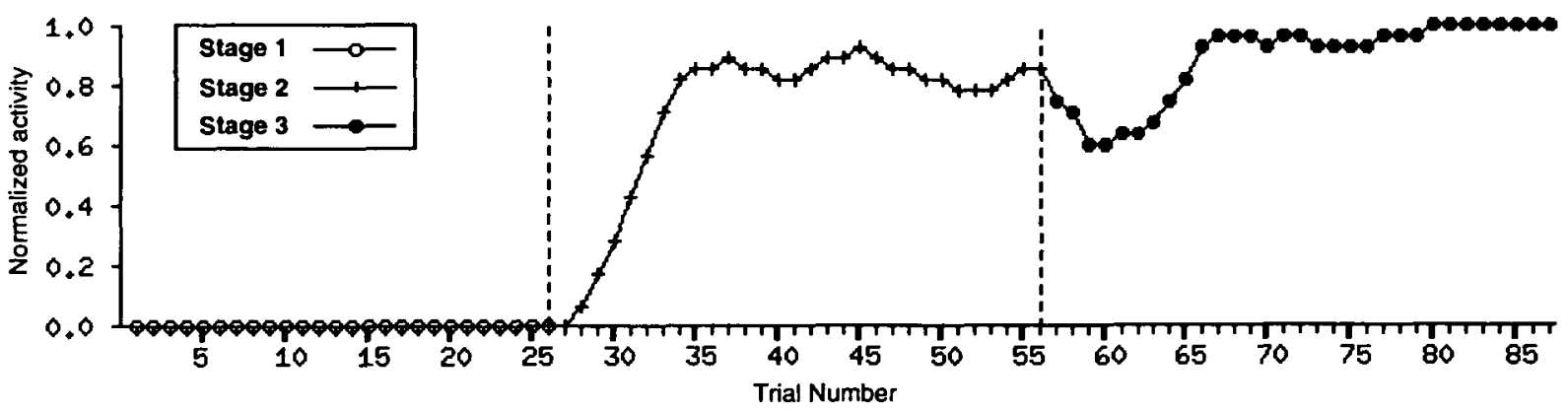

Figure 6. Variations in the activity of bistable groups (A-C) during the training period. Graphs are constructed from the activity during reinforced left trials of all the units in a group (four units per group). Trial activity is computed as follows. (1) For each unit, the unit trial activity is 1 if the unit has been made active with a probability different from 0.5 during the trial; otherwise it is 0 . (2) Trial group activity is the average unit trial activity of all the units. (3) Trial activity is obtained as the five-point left-moving averages of trial group activities and normalized. Each horizontal division corresponds to a trial. Vertical dashed lines indicate the transitions between training stages. Note the combination of increasing and decreasing activity: activity decreases at the transition between two stages and increases after the transition. Activity in group $b$, started at the second stage due to the connections to the matching units (see Fig. 2).

active when the go signal was introduced in the second training stage. The unit was nondifferentially activated by the go signal at stage 2 and responded preferentially to the go signal in left versus right trials at stage 3 . Differential activity related to the instruction stimuli was found in unit $m_{1}$ at stage 3 .

Units similar to $m_{3}$ have been found experimentally. In fact, Okano and Tanji (1987) and Romo and Schultz (1987) have observed that similar activity precedes self-initiated and visually triggered arm movements in some units, in the motor, premotor, and supplementary motor cortex.

\section{Bistable Units}

Changes in bistable units are shown in Figure 5. The units displayed sustained activities (more than two time steps of the simulation) and became differentially active for left versus right trials when a particular sensory or motor event was introduced (movement in Fig. 5A,B; go signal in Fig. 5O). The type of activity varied greatly.

The units shown in Figure $5 A$ have a behavior very similar to movement-related matching units (Fig. 4). They were active before the movement in the three training stages and were selective for right versus left trials. Figure $5 B$ shows the evolution of activity in delay-related units. These units changed their time of activation with learning (Fig. 5B, left trials). In the first stage, they started their activity with the drive and stopped it with the movement. At stage 2 , activity started with the drive but ended with go signal. In the last stage, activity started with the instruction stimulus and ended with the go signal. The units in Figure $5 \mathrm{C}$ always started their activity with the drive, but changed the time of return to rest (with the go signal at stage 2, and with the instruction stimulus at stage 3).

Changes in the level of activity in bistable groups are shown in Figure 6. Modulations are correlated with the changes in the reinforcement contingency, depending on variations in the reinforcement rate. When changing from stage 1 to stage 2, two different behaviors were alternatively performed by the network, in a way similar to that observed in monkeys: the previous correct behavior (self-initiated movements) and the new 
Figure 7. Internal representation of sequences. Activities in matching units $m_{1}$ $m_{3}, m_{3}$ (light shading) and bistable units $b_{1}, b_{3}, m_{3}$ (dark shading) are qualitatively displayed. Note the progressive differentiation of activity in the successive stages. Activities in matching units are correlated with the end of activity in bistable units.

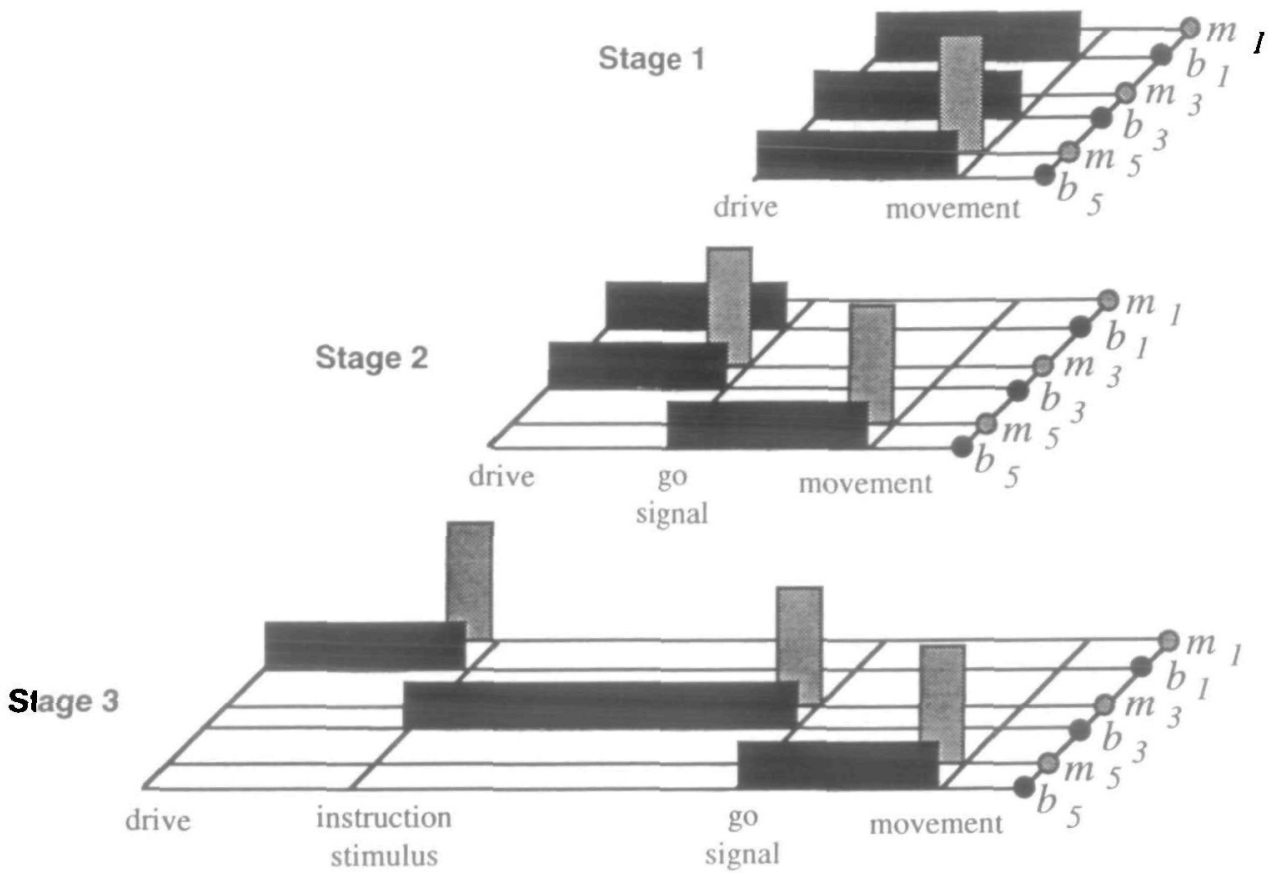

correct behavior (stimulus-triggered movements). Mean activity during reinforced trials in group $b_{5}$ increased for leftward selfinitiated movements during the first stage (open circles, Fig. $6 A$ ). During the transition between stage 1 and stage 2 , activity first decreased and increased again (crosses, Fig. 6A) with the increase in the performance (see Fig. 3). The same phenomenon was observed between stage 2 and stage 3 (solid circles, Fig. 6A).

The variations in activity of group $b_{3}$ are shown in Figure $6 B$. These variations were quite similar to those in Figure $6 A$, with monotonic (increase) changes at each stage and nonmonotonic changes (decrease and increase) at the transition between two stages. Figure $6 \mathrm{C}$ illustrates the variations in group $b_{1}$ units. In this case, variations started at stage 2 but followed the same principle.

During reversal learning in a go/no-go discrimination task, Niki et al. (1990) found some neurons that reversed their activity with the change in the reward contingency (type 2), and some that retained the same activity (type 1). The matching units of this model resemble the type 1 units since both were related to the impending behavioral response and did not change their activity with change in the reward contingency. Type 2 units (Niki et al., 1990) reversed their activity with the change in the reward contingency as do bistable units in the network. Although few units were studied, type 2 units were rarely found in the premotor cortex, while the PFC contained both types of units in similar proportions (Niki et al., 1990) This supports our hypothesis that some units (bistable units) are specific to the PFC. Changes in the activity of bistable units can also be compared with those found by Thorpe et al. (1983). Neurons in the orbitofrontal cortex decrease or increase their activity depending on learning of the associative significance of a visual stimulus. The results are also consistent with the observations of Watanabe (1990), who found decreasing activity when changing the significance of a stimulus without changing the required behavioral response. Modulations of activity in bistable units may thus be related to the change in the associative significance of the current behavior (will it lead to a reward?) (Thorpe et al., 1983; Watanabe, 1990).

The nonmonotonic changes in bistable units subserve both the development of new behaviors and the transition between two behaviors on the basis of changes in reward. Decreasing activity in response to unrewarded actions suppresses these actions. Subsequent increases in neuronal activity reflect the construction of a new rewarded behavior (e.g., the integration of new environmental cues). This view is consistent with the proposals of Fuster (1988). Indeed, Fuster (1988) suggested that newness and complexity are the main challenges to prefrontal functions. The properties of prefrontal units in the model reflect both the ability to take into account changing environmental demands and the ability to integrate a set of sensory, motor and more complex events into a coherent goal-directed behavior

\section{Internal Representation of Sequences}

The model suggests how sensorimotor programs can be represented in a neural network. The activity patterns of bistable and matching units reflect the storage of the program, as illustrated in Figure 7. Each graph corresponds to a training stage and is made from Figures 4 and 5 . It shows that task events are indicated by matching unit activities, that bistable units are active at each training stage between two successive task events, that this relationship changes from stage to stage, and that bistable units gradually become specialized for different successions of events. At stage 3, each unit is linked to a specific succession of events (drive/instruction stimulus in $b_{1}$, instruction stimulus/go signal in $b_{3}$, go signal/movement in $b_{5}$ ). Note the changing role of unit $b_{3}$ in the course of learning.

\section{Discussion}

The Delayed Response Task and Prefrontal Functions This work has focused on the functional properties of prefrontal circuits that enable a DR task to be learned. The DR task has been widely used to test cognitive aspects of prefrontal functions ever since the pioneering studies of Jacobsen (1935) Although there are still large discrepancies in our knowledge of the critical parameters that actually tap the PFC during delayed tasks (Rosenkilde, 1979), lesion studies have emphasized the importance of the temporal factor. Monkeys with bilateral prefrontal lesions are severely impaired when long intratrial delays are used (Fuster, 1988). As a general rule, a frontal le- 
sioned monkey fails to execute a behavior determined by some recent events. Convincing evidence for the involvement of PFC in DR tasks has also been obtained from electrophysiological studies. Recordings of single-cell activity have shown that neuronal discharges are stronghy correlated with task events and delays (Fuster, 1973; see review in Fuster, 1988). Thus, the DR task appears to be well suited to examining prefrontal functions. Hence, a model of the DR task offers a direct way to assess computational processes with respect to the data available on the PFC.

\section{Modeling Otber Tasks}

A number of tasks have been used to study the PFC areas in monkeys. The best known are the delayed response (DR) task, the delayed alternation (DA) task, the delayed matching to sample (DMS) task, and go/no-go task response. It is possible that all these tasks involve similar prefrontal functions. The electrophysiological properties of prefrontal neurons are relatively constant through the different protocols used, in the sense that the same set of stereotyped activities are found (Komatsu, 1982). This result is probably due to the fact that a set of common elementary processes underlie the execution of each task: expectation of environmental stimuli, short-term memorization, preparation of response, reward. This response is not completely satisfactory since it does not take into account the behavioral aspects of these mechanisms. For instance, DR and DA tasks are somewhat different; one is controlled by external cues whereas the other depends on internal cues. However, similar activities are observed in both tasks (Kubota and Niki, 1971; Niki and Watanabe, 1976). The model suggests that a single function may be used in both cases. This function could be a general ability to determine "behavior significance" (which response?) and "associative significance" (will it lead to a reward?) of a situation. In this form, the function is independent of particular modalities. It works for visual or auditory signals, with internal or external signals. Neuronal activities reflecting behavioral significance have been frequently observed, for example, differential delay activity (Niki and Watanabe, 1976) and differential activity on go and no-go trials (Komatsu, 1982). Watanabe (1990) found a strong neuronal correlation with associative significance. Monkeys were simultaneously involved in two tasks: a direct task and a corresponding reversal task. Some neurons responded differentially to the same first stimulus if the monkey was currently executing a block of direct or reversal trials (Watanabe, 1990). The response of these neurons is not determined by the physical properties of the stimulus but by the associative value of the stimulus.

Lesions studies indicate the areas of the PFC that are involved in different behavioral tasks (Rosenkilde, 1979). A prediction of the model is that anatomical specializations are related to the same set of mechanisms expressed on different architectural substrates (different patterns of corticocortical and subcortical connections). We can thus reliably assume that so-called more complex tasks would rely on more integrated signals or combinations of signals rather than on more complex functional mechanisms. Such signals have been found experimentally in several studies (Rosenkilde et al., 1981; Watanabe, 1989, 1990). Watanabe (1989) described posttrial neuronal responses linked to the correct execution of a trial, independent of external assessment (reward). In the same way as the model can capture temporal relationships between sensory and motor signals, it can capture relationships between more "abstract" signals.

\section{Extension of the Model}

The model illustrates a process that allows sequences of sensory and motor events to be stored and retrieved in a neuronal substrate. The DR task is composed of two sequences of three events. According to the model, the task can be stored using six classes of bistable units and six matching units (we have not included units corresponding to interfacing events; Fig. 2). After learning, each matching unit is dedicated to the representation of an event and each bistable class to the representation of the succession of two events. In the following, we address issues concerning the generality of this process.

It is important to note that there are no a priori representations of sequences in the network, that would lead to a combinatorial explosion. Sequences are represented by a distributed set of bistable units. The properties of these units are not predefined, but specified in the course of learning, according to the requirements of the training protocol. The model shows how such units can be reallocated when the task is made more complex. (1) Before learning, these units have a set of connections with matching units that are selective for sensory and motor events. Such connections do not store the computational demand linked with each sensory or motor events (already represented in association areas) but only their possible participation in a learned behavioral sequence. (2) Since bistable units are adaptive devices that learn the control of their input pathways and their sustained activation, the ability of the network to adapt to new requirements does not depend on a particular and unique connectivity.

Two properties can be derived from these remarks. (1) Several networks with different patterns of interconnections can learn the same task and reach the same level of performance. Differences between these networks could be found at the level of single-unit activity. However, the variations of activity corresponding to the stage-by-stage learning of the task will follow the principle shown in Figure 6 (i.e., a combination of monotonic and nonmonotonic variations related to the training steps). (2) The same network can learn different tasks, depending on the nature of information in input and output pathways and on the training protocol.

When several sequences are to be learned by the same network, the model hypothesizes that they will be stored in different groups of bistable units (semidistributed coding). Consequently, the minimal number of allocated neurons should increase linearly with both the number and the size of learned sequences. In such model, the storage capacity is directly linked with the number of neurons. Since several sensory and motor events can be shared by different sequences, a competition process could be added in the bistable layer to facilitate the learning process when more and more information is available.

\section{Rule-Coding Units-Higher-Level Modeling}

The model relies on the fact that task requirements (memorization, behavioral responses) are acquired by a set of processing units through sensorimotor experiences. Alternatively, some models employ a direct representation of task requirements by providing "rule-coding" units (Dehaene and Changeux, 1989, 1991). Dehaene and Changeux (1989) showed that the rule guiding the correct behavior of a network can be selected by learning. Thus, after learning, the activity of rule-coding units reflects the effective rule that leads to the reward. Incorrect rule selection leads to failure of the behavior. Cohen and Servan-Schreiber (1992) used similar units to learn psychological tasks in a recurrent neural network. This approach clearly simplifies computational processes, since the network is directly supervised by high-level representations of modality or programs.

Although rule-coding units are attractive for modeling $\mathrm{cog}$ nitive tasks, rules such as "choose color," "choose position" (Dehaene and Changeux, 1989, 1991; Cohen and Servan-Schreiber, 1992) appear to be not very realistic at the neuronal level. The activity of rule-coding units is more likely to express relation- 
ships such as "choose this program because it is surely rewarded" (see Watanabe, 1989, 1990).

\section{Biological Basis for the Bistable Rule}

The main hypothesis of the model is that the prefrontal cortex mediates cross-temporal contingencies via patterns of sustained activity. The origin of these sustained activities is a major question. Recurrent circuits of excitatory and inhibitory neurons are likely to produce a sustained activity (recurrent excitatory pathways) and to stop it (inhibitory pathways) (Dehaene and Changeux, 1991; Zipser, 1991). Kirillov et al. (1993) have shown that bistable behavior can be generated and precisely controlled by synaptic inputs in a two-neuron inhibitory-feedback circuit. Zipser et al. (1993) provided direct evidence for bistability of cortical neurons in a recurrent neural network trained to mimic the input-output characteristics of an active memory module.

Such circuits are likely to exist in the brain. Reciprocal connections between the dorsolateral prefrontal cortex (DLC) and the mediodorsal (MD) nucleus of the thalamus are a possible pathway (Fuster, 1988). Cooling a large portion of the DLC results in a drop in the firing frequency (during the delay) of neurons in the parvocellular portion of the MD nucleus (Fuster and Alexander, 1973). An alternative pathway is the corticostriato-pallido-thalamo-cortical loop (Alexander et al., 1986). Sustained activations during delays occur throughout the major parts of the cortico-basal ganglia loops, such as the supplementary motor area (Tanji et al., 1980), postolateral putamen (Alexander and Crutcher, 1990), pallidum (Nambu et al., 1990), and pars reticulata of substantia nigra (Hikosaka and Wurtz, 1983; Schultz, 1986). Thus, delay-related activations could be built up through successive reverberations in loops linking the cortex and the basal ganglia.

Alternatively, sustained activity may be controlled at the single-cell level, via the properties of specific ionic channels. Intrinsically generated plateau potentials have been observed in neurons from structures such as the spinal cord (Hounsgaard et al., 1984), striatum (Kawagushi et al., 1989), and the PFC (Hammond and Crépel, 1992). The plateau potential is generated by a slowly inactivating or noninactivating inward current. The plateau can be conditional and induced by a change in the balance between outward and inward currents. A slowly inactivating potassium current has been found in prefrontal neurons (Hammond and Crépel, 1992). Since this current is partly inactivated at steady-state potential, hyperpolarizing or depolarizing the neuron changes the availability of the channel and allows control of the firing mode of the neuron (Hammond and Crépel, 1992). It is also sensitive to the sequences of activation of the neuron. Prolonged near-threshold depolarizing stimuli activate this current, which initially inhibits spike firing since it overwhelms inward currents. Delayed firing is then observed, which can last for several seconds. Inactivation of the current unmasks sodium-mediated inward currents and thus allows the discharge of the neuron. Repetitive long depolarizing pulses induce a gradual reduction in the delayed activation and can favor spontaneous plateau depolarizations lasting for several seconds.

The model implies that control of the bistable behavior can be modulated by learning. First, the model considers that learning occurs through combined long-term potentiation (LTP) and long-term depression (LTD) phenomena. LTP and LTD are colocalized in the PFC of the rat (Hirsch and Crépel, 1991). Artola et al (1990) have observed that induction of LTP and LTD in the visual cortex depends on different voltage-dependent thresholds. Stimulations occurring at different levels of postsynaptic depolarization are likely to have different actions (LTP or LTD). In the model of prefrontal neurons, we have proposed that LTP and LTD processes depend on the temporal order of inputs. Second, the main effect of changing synaptic weights is to modify the probability of triggering a plateau potential and sustained neuron activity. An increased synaptic weight can facilitate the activation of persistent inward currents and the inactivation of slowly inactivating outward currents. Such an hypothesis has not been experimentally tested and must, as yet, be considered as a prediction.

\section{Prefrontal versus Sensory-Motor Processing}

One way to compare the two types of processing is to ask what kind of code is used in the different regions. Georgopoulos et al. (1982) have shown that neurons in the motor cortex are preferentially active for a given direction of arm movement (with a broad tuning around the preferred direction) and that the direction of an instructed forthcoming movement is predicted by a population code. Similarly, graded neuronal responses have been observed for saccade representations in the superior colliculus (Lee et al., 1988) and for face representation in the inferotemporal cortex (Young and Yamane, 1992). On the other hand, no simple code has been found in prefrontal neurons. The different types of neuronal activity described in the PFC are diffusely distributed and intermixed. Why is there such a redundant representation? Due to neuronal variability, no individual neuron can perform as well as a monkey during a given task (e.g., be active every time the monkey performs a given action). However, the average response of a few cells may reduce the variability of responses and provide a reliable prediction of the behavior. The present model points to a different (but not incompatible) view, that two bistable units, which show similar responses at a given training stage, are likely to become differentiated at the following stage, when the behavior becomes dependent on new conditions. In this case, the redundant representation is linked to the ability to perform new behaviors.

\section{Appendix}

\section{Notations}

$\mathbf{N}$ is the set of integer numbers, $\{0,1\}$ the set of 0 and $1, \mathbf{R}$ the set of real numbers, $\mathbf{R}^{+}$the set of non-negative real numbers, $\mathbf{R}^{+}$the set of strictly non-negative real numbers, and $[0,1]$ the closed real interval. $n \in \mathbf{N}$ is the number of input pathways; $t$ $\epsilon \mathbf{N}$ is an increasing variable describing the time step of the simulations. For each $i, 1 \leq i \leq n, x(t) \in\{0,1\}$ is the input signal in the pathway $i$. For each $i, 1 \leq i \leq n, w(t) \in[0,1]$ is the synaptic weight of the pathway $i . y(t) \in\{0,1\}$ is the output signal; $r(t) \in(0,1)$ is the reinforcement signal.

\section{Bistable Rule}

The dynamics of bistable units (Fig. 1) are defined by

$$
\begin{aligned}
y(t)= & {[1-y(t-1)] F\left[\sum_{i=1}^{n} w_{i}(t) x_{i}(t)\right] } \\
& +F[\eta y(t-1)]\left\{1-F\left[\sum_{i=1}^{n} x_{i}(t)\right]\right\},
\end{aligned}
$$

where $F$ is the stochastic function defined by

$$
F(u)= \begin{cases}1 & \text { with prob } f(u) \\ 0 & \text { with prob } 1-f(u)\end{cases}
$$

and $f$ is the function defined by

$$
f(u)= \begin{cases}0, & 0 \leq u \leq \lambda \\ \frac{u-\lambda}{\mu-\lambda}, & \lambda<u<\mu ; \\ 1, & \mu \leq u \leq 1\end{cases}
$$


$\eta, \lambda$, and $\mu$ are parameters. The function $F$ corresponds to the stochastic behavior of neurons and the function $f$ to the nonlinear relationship between membrane potential and firing. Equation 1 implies that the unit has two states of activity $(0$ and 1) and switches between these states with a probability defined by synaptic inputs (Fig. $1 B, C$ ). The first term of the equation indicates that the unit becomes active when it was previously inactive and when the synaptic inputs are sufficient. The second term indicates that the unit can stay active for a while and returns to rest following subsequent inputs. Synaptic weights vary with time from the initial value $w_{0} \in[0,1]$. They are modified according to

$$
\Delta w_{i}(t, t+1)=e_{i}(t)\left\{\sum_{j=1, j \neq i}^{n}\left[-\alpha \Delta_{i j}^{-}(t)+\beta \Delta_{i j}^{+}(t)\right]\right\},
$$

where

$$
\left\{\begin{array}{l}
\Delta_{i j}^{-}(t)=K_{i}^{-}(t) x_{j}(t) y(t-1) \\
\Delta_{i j}^{+}(t)=K_{i}^{+}(t) \bar{x}_{j}(t) \bar{y}(t) r(t)
\end{array}\right.
$$

and $\alpha$ and $\beta$ are parameters in $\mathbf{R}^{+}$; functions $K_{i}^{+}$and $K_{i}^{-}$ensure that synaptic weights vary smoothly between 0 and 1 :

$$
\left\{\begin{array}{l}
K_{i}^{+}(t)=\gamma r(t)-w_{i}(t) \\
K_{i}^{-}(t)=w_{i}(t)
\end{array}\right.
$$

and, using the notations of Sutton and Barto (1981), $\bar{x}_{i}$ is the nonconditional input trace in pathway $i$ defined by

$$
\bar{x}_{i}(t+1)=\kappa \bar{x}_{i}(t)+x_{i}(t)
$$

$e_{i}$, the conditional input trace in pathway $i$ defined by

$$
e_{i}(t+1)=\omega e_{i}(t)+x_{i}(t) y(t)
$$

$\bar{y}$, the output trace defined by

$$
\bar{y}(t+1)=\chi_{1} \bar{y}(t)+\chi_{2} y(t)[y(t)-y(t+1)] ;
$$

and $\kappa, \omega_{1} \chi_{1}$, and $\chi_{2}$ are parameters in $[0,1]$.

Equation 2 is the quantitative description of the variations of weight shown in Figure $1 B$. It indicates that the synaptic weight $w_{i}$ decreases when the sustained activity of the unit triggered by the pathway $i$ is stopped by some other pathways $\left(-\Delta_{t j}^{-}\right)$, and increases when a reinforcement signal occurs after the sustained activity $\left(\Delta_{i j}^{+}\right)$.

\section{Matching Rule}

The dynamics of matching units are defined by

$$
y(t)=g\left(\sum_{i=1}^{n} \sum_{j>1}^{n} x_{i} x_{j}(t)\right),
$$

where $g$ is the function defined by

$$
g(u)= \begin{cases}0 & \text { if } u=0 \\ 1 & \text { otherwise }\end{cases}
$$

\section{Description of the Simulations}

\section{Training Stages}

The number of trials during each stage was, for stage 1, 15 blocks of 8 trials; stages $\left(1^{\prime}+2\right), 15$ blocks of 8 trials; and stages $\left(2^{\prime}+3\right), 17$ blocks of 8 trials.

\section{Numerical Parameters}

For the simulations, the following parameter values were used: $\delta=8$ (delay length); $\lambda=0.25 ; \mu=1.0$ (transfer function); $k$ $=0.995 ; \omega=0.995 ; \chi_{1}=0.97 ; \chi_{2}=0.9$ (traces) $\eta=0.985 ; \alpha$ $=0.1 ; \beta=0.35 ; \gamma=1.0 ; w_{0}=0.5$.

\section{Sensitivity to Parameters}

The ability of the network depends on the value of the different parameters of the model, described below.

Decay rate of sustained activity $(\eta)$ defines the duration of the sustained activity. A large delay rate will mainly impair stage 3 of the training protocol, when a delay is imposed between the instruction stimulus and the go signal. However, the real effect is dependent on the redundancy of bistable units.

Learning rule parameters ( $\alpha$ and $\beta$ ). The ratio $I=\alpha / \beta$ defines the respective importance of nonreinforced and reinforced behaviors, respectively. If $I$ is low, the synaptic weights change with correct behaviors. In this case, incorrect behaviors slowly disappear. On the other hand, if $I$ is high, incorrect behaviors rapidly extinguish.

Trace parameters $(\kappa, \omega, \lambda$, and $\mu$ ) define the amplitude and the duration of activation of variables used to link successive events. If traces are too short, the behavior of the network will stay at or near random since the bistable units will not benefit from the reinforcement.

\section{Notes}

This work is supported by Grant Human Frontier Science Program ISCC, MRT Cogniscience 91COG47. We are especially thankful to June Hirsch, Francis Crépel, Xavier Mondon, Constance Hammond, and Simon Thorpe for their useful ideas and suggestions concerning this study, and to Owen Parkes and Susan Sara for revising the English.

Correspondence should be addressed to Emmanuel Guigon, INSERM CREARE, Boîte 23, Université P. et M. Curie, 9 quai St Bernard, Bât. C, Gème étage, 75005 Paris, France.

\section{References}

Alexander GE, Crutcher MD (1990) Preparation for movement: neural representations of intended direction in three motor areas of the monkey. J Neurophysiol 64:133-150.

Alexander GE, DeLong, MR, Strick PL (1986) Parallel organization of functionally segregated circuits linking basal ganglia and cortex. Annu Rev Neurosci 9:357-381.

Andersen RA, Essick GK, Siegel RM (1987) Neurons of area 7 activated by both visual stimuli and oculomotor behavior. Exp Brain Res 67: 316-322.

Artola A, Bröcher S, Singer W (1990) Different voltage-dependent thresholds for inducing long-term depression and long-term potentiation in slices of rat visual cortex. Nature 347:69-72.

Barbas H, Mesulam MM (1985) Cortical afferent input of the principalis region of the rhesus monkey. Neuroscience 15:619-637.

Barbas H, Pandya DN (1987) Architecture and frontal cortical connections of the premotor cortex (area 6 ) in the rhesus monkey.J Comp Neurol 256:211-228.

Batuev AS, Shaefer VI, Orlov AA (1985) Comparative characteristics of unit activity in the prefrontal and parietal areas during delayed performance in monkeys. Behav Brain Res 16:57-70.

Bienenstock EL, Cooper LN, Munro PW (1982) Theory for the development of neuron selectivity: orientation specificity and binocular interaction in visual cortex. J Neurosci 2:32-48.

Burnod Y, Grandguillaume P, Otto I, Ferraina S, Johnson PB, Caminiti R (1992) Visuo-motor transformations underlying arm movements toward visual targets: a neural network model of cerebral cortical operations. J Neurosci 12:1435-1453.

Caminiti R, Johnson PB, Galli C, Ferraina S, Burnod Y (1991) Making arm movements within different parts of space: the premotor and motor cortical representation of a coordinate system for reaching to visual targets. J Neurosci 11:1182-1197.

Cavada C, Goldman-Rakic PS (1989) Posterior parietal cortex in rhesus monkey. II. Evidence for segregated cortico-cortical networks linking sensory and limbic areas with the frontal lobe. J Comp Neurol 287:422-445.

Cohen JD, Servan-Schreiber D (1992) Context, cortex, and dopamine: a connectionist approach to behavior and biology in schizophrenia. Psychol Rev 99:45-77.

Dehaene S, Changeux JP (1989) A simple model of prefrontal cortex function in delayed-response tasks. J Cognit Neurosci 1:244-261.

Dehaene S, Changeux JP (1991) The Wisconsin Card Sorting Test: 
theoretical analysis and modeling in a neuronal network. Cereb Cortex 1:62-79.

di Pellegrino G, Wise SP (1991) A neurophysiological comparison of three distinct regions of the primate frontal lobe. Brain 114:951978.

Eskandar EN, Richmond BJ, Optican LM (1992a) Role of inferior temporal neurons in visual memory. I. Temporal encoding of information about visual images, recalled images, and behavioral context. J Neurophysiol 68:1277-1295.

Eskandar EN, Richmond BJ, Optican LM (1992b) Role of inferior temporal neurons in visual memory. II. Multiplying temporal waveforms related to vision and memory. J Neurophysiol 68:1296-1306.

Funahashi S, Bruce CJ, Goldman-Rakic PS (1990) Visuospatial coding in primate prefrontal neurons revealed by oculomotor paradigms J Neurophysiol 63:815-831.

Fuster JM (1973) Unit activity in prefrontal cortex during delayedresponse performance: neuronal correlates of transient memory. J Neurophysiol 36:61-78.

Fuster JM (1988) The prefrontal cortex, 2d ed. New York: Raven.

Fuster JM, Alexander GE (1973) Firing changes in cells of the nucleus medialis dorsalis associated with delayed response behavior. Brain Res 61:79-91.

Fuster JM, Bauer RH, Jervey JP (1985) Functional interactions between inferotemporal and prefrontal cortex in a cognitive task. Brain Res 330:299-307.

Georgopoulos AP, Kalaska JF, Caminiti R, Massey JT (1982) On the relations between the direction of two-dimensional arm movements and cell discharge in primate motor cortex. J Neurosci 2: 1527-1537.

Georgopoulos AP, Schwartz AB, Kettner RE (1986) Neuronal population coding of movement direction. Science 233:1416-1419.

Goldman-Rakic PS (1987) Circuitry of primate prefrontal cortex and regulation of behavior by representational memory. In: Handbook of physiology, Sec 1, The nervous system, Vol 5, Higher functions of the brain, Chap 9 (Plum F, ed), pp 373-417. Bethesda, MD: American Physiological Society.

Hammond C, Crépel F (1992) Evidence for a slowly inactivating $\mathrm{K}^{+}$ current in prefrontal cortical cells. Eur J Neurosci 4:1087-1092.

Hebb DO (1949) The organization of behaviour. New York: Wiley

Hertz JA, Palmer RG, Krogh AS (1991) Introduction to the theory of neural computation. Reading: Addison-Wesley.

Hikosaka O, Wurtz HR (1983) Visual and oculomotor functions of monkey substantia nigra pars reticulata. III. Memory-contingent visual and saccade responses. J Neurophysiol 49:1268-1284.

Hirsch JC, Crépel F (1991) Blockade of NMDA receptors unmasks a long-term depression in synaptic efficacity in rat prefrontal neurons in vitro. Exp Brain Res 85:621-624.

Hounsgaard J, Hultborn H, Jespersen B, Kiehn O (1984) Intrinsic membrane properties causing a bistable behavior of $\alpha$-motoneurones Exp Brain Res 55:391-394.

Hubel DH, Wiesel TN (1968) Receptive fields and functional architecture of monkey striate cortex. J Physiol (Lond) 195:215-243.

Jacobsen CF (1935) Functions of frontal association areas in primates. Arch Neurol Psychiatry 33:558-569.

Johnson PB (1992) Toward an understanding of the cerebral cortex and reaching movements: a review of recent approaches. In: Control of arm movement in space. Neurophysiological and computational approaches (Caminiti R, Johnson PB, Burnod Y, eds), pp 199261. Heidelberg: Springer.

Jones EG (1969) Interrelationship of parieto-temporal and frontal cortex in the rhesus monkey. Brain Res 13:412-415.

Jones EG, Powell TPS (1970) An anatomical study of converging sensory pathways within the cerebral cortex of the monkey. Brain 93: $793-820$

Kawagushi Y, Wilson CJ, Emson PC (1989) Intracellular recording of identified neostriatal patch and matrix spiny cells in a slice preparation preserving cortical inputs. J Neurophysiol 62:1052-1068.

Kiehn $O$ (1991) Plateau potentials and active integration in the final common pathway for motor behavior. Trends Neurosci 14:68-73.

Kirillov AB, Myre CD, Woodward DJ (1993) Bistability, switches and working memory in a two-neuron inhibitory-feedback model. Biol Cybern 68:441-449.

Kohonen $T$ (1988) Selforganization and associative memory. Berlin: Springer.

Kojima S, Goldman-Rakic PS (1982) Delay-related activity of prefrontal neurons in thesus monkeys performing delayed response. Brain Res 248:43-49.

Komatsu H (1982) Prefrontal unit activity during a color discrimination task with GO and NO-GO responses in the monkey. Brain Res 244:269-277.

Kubota K, Komatsu S (1985) Neuron activities of monkey prefrontal cortex during learning of visual discrimination tasks with $\mathrm{GO} / \mathrm{NO}$ GO performances. Neurosci Res 3:106-129.

Kubota K, Niki H (1971) Prefrontal cortical unit activity and delayed alternation performance in monkeys. $J$ Neurophysiol 34:337-347.

Lee C, Rohrer WH, Sparks DL (1988) Population coding of saccadic eye movements by neurons in the superior colliculus. Nature 332 357-360.

Ljungberg T, Apicella P, Schultz. W (1992) Responses of monkey dopamine neurons during learning of behavioral reactions. $J$ Neurophysiol 67:145-163

Maunsell JHR, Newsome WT (1987) Visual processing in monkey ex trastriate cortex. Annu Rev Neurosci 10:363-401.

McCulloch WS, Pitts WH (1943) A logical calculus of the ideas immanent in nervous activity. Bull Math Biophys 5:115-133.

McGinty D, Szymusiak R (1988) Neuronal activity patterns in behaving animals: brainstem and limbic system. Annu Rev Psychol 39:135168.

Mikami A, Kubota K (1980) Inferotemporal neuron activities and color discrimination with delay. Brain Res 182:65-78.

Mishkin M, Ungerleider LG, Macko KA (1983) Object vision and spatial vision: two cortical pathways. Trends Neurosci 6:414-417.

Nambu A, Yoshida S, Jinnai K (1990) Discharge patterns of pallidal neurons with input from various cortical areas during movement in the monkey. Brain Res 519:183-191.

Niki H, Watanabe M (1976) Prefrontal unit activity and delayed re sponse: relation to cue location versus direction response. Brain Res 105:79-88.

Niki H, Sugita S, Watanabe M (1990) Modification of the activity of primate frontal neurons during learning of a $\mathrm{GO} / \mathrm{NO}-\mathrm{GO}$ discrimination and its reversal. A progress report. In: Vision, memory, and the temporal lobe (Iwai E, Mishkin M, eds), pp 305-311. New York: Elsevier.

Okano K, Tanji J (1987) Neuronal activities in the primate motor fields of the agranular frontal cortex preceding visually triggered and selfpaced movements. Exp Brain Res 66:155-166.

Petrides M, Pandya DN (1984) Projections to the frontal cortex from the posterior parietal region in the rhesus monkey. J Comp Neurol 228: 105-116.

Rolls ET (1990) The representation of information in the tempora lobe visual cortical areas of macaques. In: Advanced neural computers (Eckmiller R, ed), pp 69-78. Amsterdam: Elsevier.

Romo R, Schultz W (1987) Neuronal activity preceding self-initiated or externally timed arm movements in area 6 of monkey cortex. Exp Brain Res 67:656-662.

Rosenkilde CE (1979) Functional heterogeneity of the prefrontal cortex in the monkey: a review. Behav Neural Biol 25:301-345.

Rosenkilde CE, Bauer RH, Fuster JM (1981) Single cell activity in ventral prefrontal cortex of behaving monkeys. Brain Res 209:375-394.

Schultz W (1986) Activity of pars reticulata neurons of monkey substantia nigra in relation to motor, sensory, and complex events. J Neurophysiol 55:660-677.

Sejnowski TJ (1986) Open questions about computation in cerebral cortex. In: Parallel distributed processing, explorations in the microstructure of cognition, Vol 2, Psychological and biological models (Feldman JA, Hayes PJ, Rumelhart DE, eds), pp 372-389. Cambridge, MA: MTT Press.

Shallice $T$ (1988) From neuropsychology to mental structure. Cambridge: Cambridge UP.

Stent GS (1973) A physiological mechanism for Hebb's postulate learning. Proc Natl Acad Sci USA 70:997-1001

Sutton RS, Barto AG (1981) Toward a modern theory of adaptive networks: expectation and prediction. Psychol Rev 88:135-170.

Tanji J, Tanigushi K, Saga T (1980) Supplementary motor area: neuron al responses to motor instructions. J Neurophysiol 43:60-68.

Thorpe SJ, Rolls ET, Maddison S (1983) The orbitofrontal cortex: neuronal activity in the behaving monkey. Exp Brain Res 49:93-115.

Toates F (1986) Motivational systems. Cambridge: Cambridge UP.

Trotter Y, Celebrini S, Stricanne B, Thorpe S, Imbert M (1992) Modu 
lation of neural stereoscopic processing in primate area $\mathrm{V} 1$ by the viewing distance. Science 257:1279-1281.

Ungerleider LG, Gaffan D, Pelak VS (1989) Projections from inferior temporal cortex to prefrontal cortex via the uncinate fascicle in rhesus monkey. Exp Brain Res 76:473-484.

Watanabe M (1986) Prefrontal unit activity during delayed conditional go/no-go discrimination in the monkey. I. Relation to the stimulus. Brain Res 382:1-14.

Watanabe $M$ (1989) The appropriateness of behavioral responses coded in post-trial activity of primate prefrontal units. Neurosci Lett 101:113-117.

Watanabe $M$ (1990) Prefrontal unit activity during associative learning in the monkey. Exp Brain Res 80:296-309.

Young MP, Yamane S (1992) Sparse population coding of faces in the inferotemporal cortex. Science 256:1327-1331.

Zipser D (1991) Recurrent network model of the neural mechanism of short-term active memory. Neural Comput 3:179-193.

Zipser D, Kehoe B, Littlewort G, Fuster J (1993) A spiking network model of short-term active memory. J Neurosci 13:3406-3420. 